\title{
Internalisation of core-shell superparamagnetic nanoparticles into human granulocytes
}

\section{F. De Angelis}

Department of Anatomy, Histology, Forensic Medicine and Orthopaedics, Sapienza University of Rome,

Via A. Borelli, 50, 00161 Rome, Italy

and

Center for Life Nano Science@Sapienza, Istituto Italiano di Tecnologia (IIT),

Viale Regina Elena, 291, 00161 Rome, Italy

Fax: +3906490631

Email: f.deangelis@uniroma1.it

\section{G. Berardi}

Department of Chemistry, Sapienza University of Rome, Piazzale Aldo Moro 5, 00185 Rome, Italy Fax: +3906 490631

Email: ginevra.berardi@uniroma1.it

\section{F.A. Scaramuzzo}

Department of Basic and Applied Sciences for Engineering, Sapienza University of Rome,

Via A. Scarpa 14/16, 00161 Rome, Italy

Fax: +39 06490631

Email: francesca.scaramuzzo@uniroma1.it

\section{Liberatore}

Department of Radiological, Oncological and Anatomo-Pathological Sciences,

Sapienza University of Rome,

Piazzale Aldo Moro 5, 00185 Rome, Italy

Fax: +3906490631

Email: maurlib@tin.it 


\title{
M. Barteri*
}

Department of Chemistry, Sapienza University of Rome, Piazzale Aldo Moro 5, 00185 Rome, Italy Fax: +39 06490631

Email: mario.barteri@uniroma1.it

${ }^{*}$ Corresponding author

\begin{abstract}
In this paper, multitasking core-shell $\mathrm{Fe}_{3} \mathrm{O}_{4} @ \mathrm{Cu} @ \mathrm{Au}$ superparamagnetic nanoparticles (MNPs) functionalised either with folic acid (FA) or methotrexate (MTX) have been used for engulfment of human granulocytes. MNPs internalisation into phagocytes allows to use the cells as carriers thus avoiding the rapid clearance and decreasing of concentration commonly experienced in case of in vivo nanoparticles administration. MNPs have been coated with poly-L-lysine (PLL), which contributes to improve their dispersion into polar solvents. Moreover, this coating improves the cellular uptake and allows the further functionalisation with MTX and FA. Experimental evidences of granulocytes engulfment have been obtained by both SEM and fluorescence microscopy, upon prior labelling of the lysines residues with suitable fluorescent probes. The superparamagnetic nature of the MNPs makes the system easily drivable into a specific tissue upon exposure to a defined magnetic field. The success of the internalisation experiments indicates that the system is a promising tool as theranostic agent for treatment of a variety of diseases, including tumours.
\end{abstract}

Keywords: core-shell magnetic nanoparticles; granulocytes; methotrexate; folic acid; nanobiotechnology.

Reference to this paper should be made as follows: De Angelis, F., Berardi, G., Scaramuzzo, F.A., Liberatore, M. and Barteri, M. (2016) 'Internalisation of core-shell superparamagnetic nanoparticles into human granulocytes', Int. J. Nanotechnol., Vol. 13, Nos. 8/9, pp.659-666.

Biographical notes: Francesca De Angelis graduated cum laude in Chemistry. She is now $\mathrm{PhD}$ student in Morphofunctional Science and Biophysics at Sapienza University of Rome, Italy. Her PhD project, funded by the Istituto Italiano di Tecnologia (IIT), deals with synthesis of nanoparticles for the theranostic of tumours.

Ginevra Berardi graduated cum laude in Chemistry and now she is $\mathrm{PhD}$ student in Chemistry at Sapienza University of Rome, Italy.

Francesca A. Scaramuzzo graduated cum laude in Chemistry at Sapienza University of Rome, Italy. She obtained her $\mathrm{PhD}$ in co-tutorship at Sapienza and at the University of Twente, The Netherlands. Later on, she was Post-Doctoral Researcher at the University of Padova, Italy. At present, she works on nanostructured materials at the Department for Basic and Applied Sciences for Engineering at Sapienza University of Rome.

Mauro Liberatore got his degree in Medicine at Sapienza University of Rome, Italy, and he is specialist in Nuclear Medicine. Teaching appointment in: (1) Nuclear Medicine; (2) Radiological Techniques; (3) Oncology. He is Member of the International Reviewers Panel of Medical Science Monitor and of the Editorial Advisory Board of the Open Nuclear Medicine Journal. 


\begin{abstract}
Mario Barteri is Full Professor of Physical Chemistry at Sapienza University of Rome, Italy. Teaching appointment in: (1) Biophysical Chemistry; (2) Chemical Kinetics; (3) Spectroscopy of Biological Systems. He implemented various projects INFN on Synchrotron Radiation facilities for studies on protein structure and in the field of nanosystems for biomedical applications. He has published more than 200 papers in peer-reviewed international journals.

This paper is a revised and expanded version of a poster entitled 'Internalization of core-shell superparamagnetic nanoparticles into granulocytes' presented at 16th Edition of Trends in Nanotechnology International Conference (TNT2015), Toulouse, France, 7-11 September, 2015.
\end{abstract}

\title{
1 Introduction
}

Iron oxide nanoparticles (IONPs) are widely used in many in vitro and in vivo biomedical applications such as MRI, hyperthermia and magnetically controlled drug delivery [1]. As such, they are considered an excellent tool to improve medical diagnosis and to achieve a targeted delivery of medications in specific tissues [2]. The widespread use of IONPs is owing to the fact that they are not toxic and their magnetic properties allow their detection, isolation and manipulation using an external magnetic field [3]. For theranostic uses it is important to optimise synthetic protocols providing samples of reduced dimensions $(5<d<40 \mathrm{~nm})$ and polydispersity. Since in aqueous solution IONPs form large aggregates [4], various synthetic methods involving the use of surfactants and polymers have been developed [5]. However, magnetite undergoes oxidation that alters its crystalline structure and consequently its superparamagnetic behaviour. To overcome this drawback, superparamagnetic core can be protected by either metallic or polymeric functional shells [6]. In this field, our group has recently been interested in the use of core-shell superparamagnetic nanoparticles consisting of a nucleus of magnetite, a first shell of $\mathrm{Cu}$ and an additional housing of $\mathrm{Au}$ properly functionalised with drugs for application as theranostic agent [7,8]. However, moving from in vitro to in vivo environment, nanoparticles (NPs) normally undergo a strong interaction with the proteins contained in the blood (i.e., albumins and opsonins), which results in the formation of aggregates and their confinement in reservoir organs such as liver, spleen or kidneys. In addition to this, white blood cells, in particular macrophages and lymphocytes, recognise NPs as non-physiological components, rapidly internalising and removing them from the blood flux [9]. The last occurrence, coupled to white blood cells' ability to be chemically attracted by tumours and inflamed tissues, suggested their use as carriers of NPs engineered with specific therapeutic agents directly to the pathological area [10]. Briefly, medicated NPs are internalised into isolated and purified phagocytes in a culture medium lacking proteins, thus avoiding the formation of protein corona, and engulfed cells are subsequently re-infused. This last procedure has been tested for both diagnostic and therapeutic purposes [11], to restrict the treatment to the affected organ and possibly to reduce the chemotherapy toxic side effects. Here, we show data obtained via different techniques which demonstrate the actual engulfment of human granulocytes with multitasking engineered superparamagnetic nanoparticles (MNPs). In detail, 
we functionalised $\mathrm{Fe}_{3} \mathrm{O}_{4} @ \mathrm{Cu} @ \mathrm{Au}$ NPs with either folic acid (FA) or its structural analogue methotrexate (MTX), normally used as chemotherapeutic drug.

\section{Materials and methods}

\section{Materials}

All the chemicals, either from Sigma-Aldrich or Fluka, were used without further purification.

Synthesis of $\mathrm{Fe}_{3} \mathrm{O}_{4} @ \mathrm{Cu} @$ Au, coating with PLL and functionalisation with FA

Performed as already described in Passeri et al. [7].

\section{Functionalisation with MTX}

Performed accordingly to the same protocol already used for functionalisation with FA [7].

\section{Labelling of eitherMNPs@FA orMNPs@MTXwith Texas Red}

6 mg of MNPs@FA or MNPs@MTX were dispersed in $6 \mathrm{~mL}$ of chilled carbonate buffer $0.1 \mathrm{M}$ at $\mathrm{pH}=9$. The reaction mixture was left react under mechanical stirring at $0^{\circ} \mathrm{C}$ for 1 h. The labelled NPs (MNPs@FA@TR or MNPs@MTX@TR) were separated from the reaction solution by magnetic decantation and washed several times with PBS buffer (20 mM PB, $150 \mathrm{mM} \mathrm{NaCl}, \mathrm{pH}=7.5$ ).

\section{Human granulocytes cell culture and incubation with functionalised MNPs}

Human granulocytes were separated from whole human blood by gradient centrifugation with Histopaque 1077 and Histopaque 1119. The granulocytes number was determined by cytofluorimetry. $16 \times 10^{6}$ granulocytes were dispersed in $850 \mu \mathrm{L}$ of protein free medium Dulbecco's modified eagle medium (DMEM) and divided in three aliquots. The first two aliquots of $350 \mu \mathrm{L}$ were incubated respectively with $650 \mu \mathrm{L}$ of a MNPs@FA@TR and 650 $\mu \mathrm{L}$ of a MNPs@MTX@TR dispersion $(1 \mathrm{mg} / \mathrm{mL}$ in PBS $\mathrm{pH}=7.4$ each). The last aliquot of $150 \mu \mathrm{L}$ was used as control and simply diluted into $800 \mu \mathrm{L}$ of $\mathrm{PBS} \mathrm{pH}=7.4$. The samples were incubated at $37^{\circ} \mathrm{C}$ in $5 \%$ $\mathrm{CO}_{2}$ either for $2 \mathrm{~h} 30^{\prime}$ or overnight. After the chosen incubation time, $500 \mu \mathrm{L}$ of each sample were centrifuged at $1200 \mathrm{rpm}$ for 10'; the pellet obtained was dispersed in $500 \mu \mathrm{L}$ of PBS and fixed for 15 ' at r.t. in $4 \%$ formalin and $4 \%$ sucrose. Subsequently the samples were centrifuged and the granulocytes pellet was dispersed in $300 \mu \mathrm{L}$ of PBS. $150 \mu \mathrm{L}$ of each sample were incubated with $15 \mu \mathrm{L}$ of Hoechst solution $(0.01 \mu \mathrm{g} / \mu \mathrm{L})$ for 15 ' at r.t. Finally, the sample was centrifuged at $1200 \mathrm{rpm}$ for 10', the cells were washed with $\mathrm{H}_{2} \mathrm{O}$, centrifuged and raised in $50 \mu \mathrm{L}$ of $\mathrm{H}_{2} \mathrm{O}$. The samples not stained with Hoechst were washed twice with $\mathrm{H}_{2} \mathrm{O}$, centrifuged at $1200 \mathrm{rpm}$ for $10^{\prime}$ and raised in $50 \mu \mathrm{L}$ of $\mathrm{H}_{2} \mathrm{O}$. Trypan-blue tests were carried out to assess viability of cells. 


\section{Results and discussion}

$\mathrm{Fe}_{3} \mathrm{O}_{4} @ \mathrm{Cu} @ \mathrm{Au}$ MNPs were synthesised by chemical co-precipitation method and characterised by different techniques such as VSM, AFM and TEM according to a procedure already optimised in our group and fully described [7]. A scheme of the MNPs is reported in Figure 1(a).

Figure 1 General scheme of core-shell $\mathrm{Fe}_{3} \mathrm{O}_{4} @ \mathrm{Cu} @ \mathrm{Au}$ nanoparticles fabrication (a) and typical VSM spectrum (b), AFM (c) and TEM (d) images (see online version for colours)

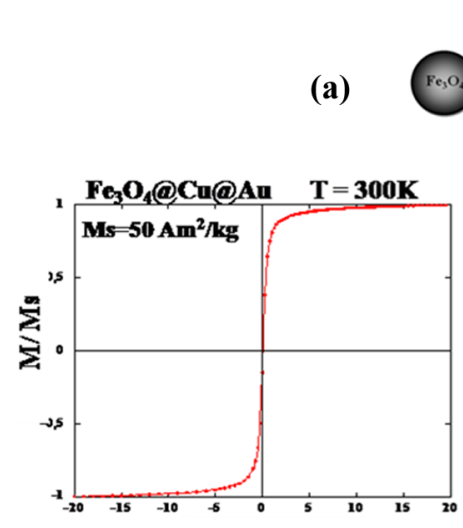

(b)
H[KOe]

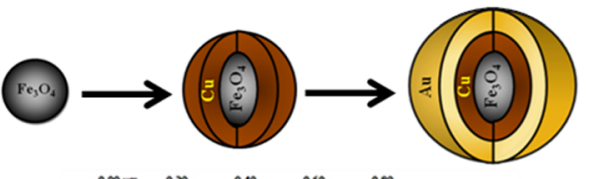

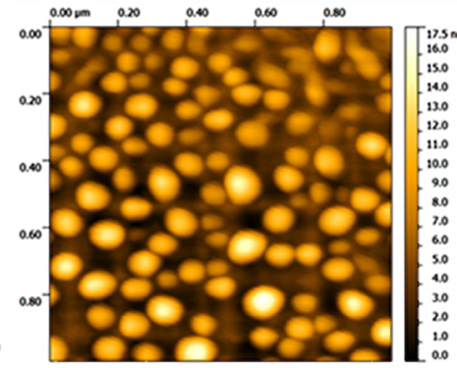

(c)

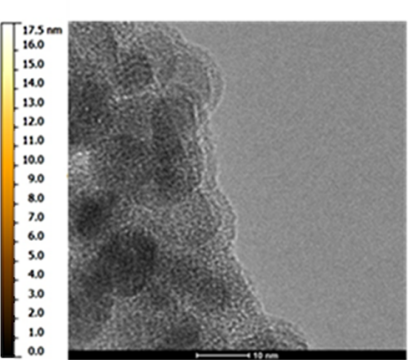

(d)

VSM (Figure 1(b)) confirmed the MNPs superparamagnetic behaviour, while AFM (Figure 1(c)) and TEM (Figure 1(d)) indicate an average size of $20 \pm 6 \mathrm{~nm}$. The magnetite core provides the superparamagnetic behaviour to the MNPs, making the system easily drivable to the target area with an external magnetic field; the $\mathrm{Cu}$ shell ensures the perfect match between magnetite and $\mathrm{Au}$, and $\mathrm{Au}$ outer coating allows functionalisation with organic molecules exploiting the well known Au-S chemistry. This core-shell nanosystem was engineered with poly-L-lysine (PLL) anchored via a covalent bond with the N-hydroxysuccinimide ester of lipoic acid, acting as a linker between the Au surface and the polymer (Figure 2).

Figure 2 Schematic representation of the NPs functionalisation reaction sequence (see online version for colours)

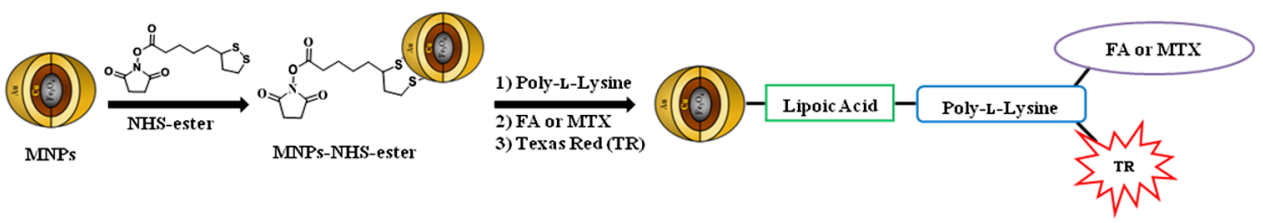

The polyelectrolytic feature of PLL improves the cellular uptake while its $\varepsilon$-amino groups allow the further functionalisation either with MTX or FA. The synthesis was performed via a one-pot reaction between the $\varepsilon$-amino groups of PLL and the N-hydroxysuccinimide ester of MTX or FA carboxylic groups. Moreover, the free residues of Lys on MNPs were labelled with the fluorescent probe Texas Red (TR). The system thus obtained, 
schematised in Figure 2, was characterised via UV-Vis, IR and fluorescence spectroscopy (data not shown) and finally used for human granulocytes engulfment.

For in vitro experiments, a population of mixed granulocytes separated from other blood components was incubated either with MNPs@FA@TR or MNPs@MTX@TR, for both $2 \mathrm{~h} \mathrm{30}$ ' and overnight, while a sample of non-treated cells was used as a reference. The former time range was chosen according to our previous studies [12], while the latter was chosen to establish if phagocytosis could be improved and to highlight possible MNPs side effects on cell physiology. The internalisation of medicated nanoparticles into the cellular target was studied by means of optical, SEM-EDX and fluorescence microscopy. Figures 3(a) and (b) show that at $2 \mathrm{~h} \mathrm{30}$ ' of incubation either with MNPs@FA@TR or MNPs@MTX@TR granulocytes keep their typical multi-lobed nuclei and average diameter of $10 \mu \mathrm{m}$ [13], while no evident changes in the cellular shape are visible. The diffuse red fluorescence surrounding the cellular nucleus derives from the TR probe anchored to the nanoparticles surface. The spatial correspondence of the blue and red fluorescence indicates the effective engulfment of the phagocytic cells with the engineered nanoparticles.

On the other hand, in the samples incubated overnight some cells show structural damages (data not shown). SEM confirms that cells treated for $2 \mathrm{~h} 30^{\prime}$ are comparable to the reference, with a spherical shape and a diameter of 5-6 $\mu \mathrm{m}$ (Figures 3(c) and (d)). The discrepancy in cell dimension obtained with the two different techniques is probably owing to dehydration occurred during sample preparation for SEM analysis. Furthermore, the EDX analysis demonstrates the presence of $\mathrm{Fe}$ and $\mathrm{Au}$ within the cells treated with the two kind of nanoparticles, while the intermediate $\mathrm{Cu}$ shell was not detected probably because of its low concentration. In the two samples, the percentage of $\mathrm{Au}$ and $\mathrm{Fe}$, normalised by their atomic weight, is comparable, going from $0.11 \%$ to $0.14 \%$ for $\mathrm{Fe}$ and from $0.01 \%$ to $0.03 \%$ for Au (Figures $3(\mathrm{e})$ and (f)). No traces of the two metals were detected in the reference sample.

Figure 3 Fluorescence microscopy and SEM images of granulocytes incubated for $2 \mathrm{~h} 30$ ' with MNPs@FA@TR (a, c) and MNPs@MTX@TR (b, d) respectively. The red colour in the cytoplasm is due to the TR probe on the NPs, while the blue one is due to the nuclear stain Hoechst 33258. The EDX analysis (e, f) demonstrates the presence of both $\mathrm{Fe}$ and $\mathrm{Au}$ (see online version for colours)
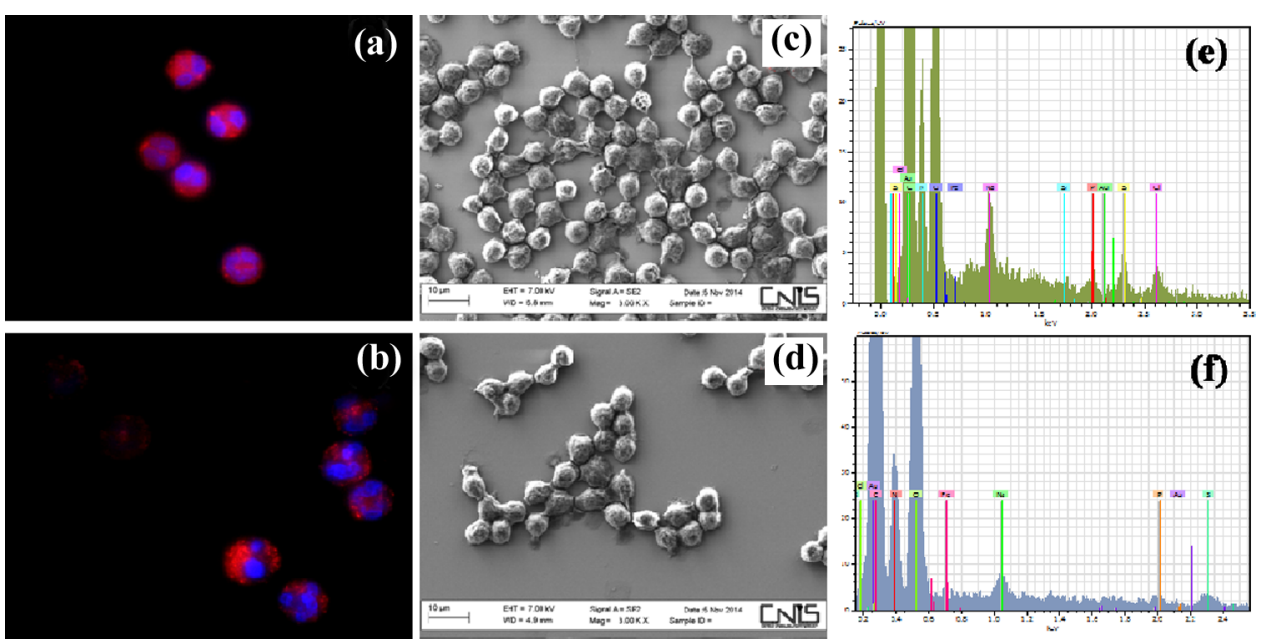


\section{Conclusions}

Magnetic core-shell nanoparticles of $\mathrm{Fe}_{3} \mathrm{O}_{4} @ \mathrm{Cu} @ \mathrm{Au}$ coated with PLL and functionalised either with MTX or FA have been successfully internalised into human granulocytes. Upon $2 \mathrm{~h} 30^{\prime}$ of incubation with the two kinds of functionalised nanoparticles, no evident cellular damages were detected. The engulfment of granulocytes paves the way to a novel approach to injuries diagnosis and therapy: exploiting the defence role of these cells and their ability to reach damaged tissues, it is possible to increase the local concentration of medicated NPs and to overcome some of the main issues concerning the in vivo applications of nanodrugs.

\section{Acknowledgements}

Dr. Francesco Mura of CNIS Sapienza for FESEM and Dr. Sergio Brutti of University of Potenza for TEM images are acknowledged. Research activity partially supported by IIT.

\section{References}

1 Kohler, N., Sun, C., Fichtenholtz, A., Gunn, J., Fang, C. and Zhang, M. (2006) 'Methotrexateimmobilized poly(ethylene glycol) magnetic nanoparticles for MR imaging and drug delivery', Small, Vol. 2, pp.785-792.

2 De, M., Ghosh, P.S. and Rotello, V.M. (2008) 'Applications of nanoparticles in biology', Adv. Mater., Vol. 20, pp.1-17.

3 Lu, A.H., Salabas, E.L. and Schüth, F. (2007) 'Magnetic nanoparticles: synthesis, protection, functionalization and application', Angew. Chem. Int. Ed., Vol. 46, pp.1222-1244.

4 Mahmed, N., Heczko, O., Söderberg, O. and Hannula, S-P. (2011) 'Room temperature synthesis of magnetite $\left(\mathrm{Fe}_{3-\delta} \mathrm{O}_{4}\right)$ nanoparticles by a simple reverse co-precipitation method', IOP Conf. Series: Materials Science and Engineering, Vol. 18, p.032020: 4 pages.

5 Zhang, Y., Liu, J-Y., Ma, S., Zhang, Y-J., Zhao, X., Zhang, X-D. and Zhang, Z-D. (2010) 'Synthesis of PVP-coated ultra-small $\mathrm{Fe}_{3-\delta} \mathrm{O}_{4}$ nanoparticles as a MRI contrast agent', J. Mater. Sci.: Mater. Med., Vol. 21, pp.1205-1210.

6 Goon, I.Y., Lai, L.M.H., Lim, M., Munroe, P., Gooding, J.J. and Amal, R. (2009) 'Fabrication and dispersion of gold-shell-protected magnetite nanoparticles: systematic control using polyethyleneimine', Chem. Mater., Vol. 21, pp.673-681.

7 Passeri, D., Dong, C., Reggente, M., Angeloni, L., Barteri, M., Scaramuzzo, F.A., De Angelis, F., Marinelli, F., Antonelli, F., Rinaldi, F., Marianecci, C., Carafa, M., Sorbo, A., Sordi, D., Arends, I.W.C.E. and Rossi, M. (2014) 'Magnetic force microscopy: quantitative issues in biomaterials', Biomatter, Vol. 4, e29507, DOI:10.4161/biom.29507.

8 Liberatore, M., Barteri, M., Megna, V., D’Elia, P., Rebonato, S., Latini, A., De Angelis, F., Scaramuzzo, F.A., De Stefano, M.E., Guadagno, N., Chondrogiannis, S., Maffione, A.M., Rubello, D., Pala, A. and Colletti, P.M. (2015) 'Special report: effect of external magnetic field on intravenous ${ }^{99 \mathrm{~m}} \mathrm{Tc}$-labelled aminosilane coated iron-oxide nanoparticles: demonstration in a rat model', Clin. Nucl. Med., Vol. 40, pp.e-104-e-110.

9 Kolaczkowska, E. and Kubes, P. (2013) 'Neutrophil recruitment and function in health and inflammation', Nat. Rev. Immunol., Vol. 13, pp.159-175.

10 Choi, M.R., Stanton-Maxey, K.J., Stanley, J.K., Levin, C.S., Bardhan, R., Akin, D., Badve, S., Sturgis, J., Robinson, J.P., Bashir, R., Halas, N.J. and Clare, S.E. (2007) 'A cellular Trojan Horse for delivery of therapeutic nanoparticles into tumors', Nano Lett., Vol. 7, pp.3759-3765. 
11 Babič, M., Schmiedtova, M., Poledne, R., Herynek, V. and Horak, D. (2015) 'In vivo monitoring of rat macrophages labeled with poly(L-lysine)-iron oxide nanoparticles', J. Biomed. Mater. Res., Part B, Vol. 103B, pp.1141-1148.

12 Pala, A., Liberatore, M., D’Elia, P., Nepi, F., Megna, V., Mastantuono, M., All-Nahhas, A., Rubello, D. and Barteri, M. (2012) 'Labelling of granulocytes by phagocytic engulfment with ${ }^{64} \mathrm{Cu}$-labelled chitosan-coated magnetic nanoparticles', Mol. Imaging Biol., Vol. 14, pp.593-598.

13 Douglas, S.D. and Ho, W-Z. (1995) Morphology of Monocytes and Macrophages, McGraw-Hill, New York. 